\title{
Next-generation sequencing of small RNAs from inner ear sensory epithelium identifies microRNAs and defines regulatory pathways
}

Anya Rudnicki ${ }^{1 \dagger}$, Ofer Isakov ${ }^{2 \dagger}$, Kathy Ushakov ${ }^{1}$, Shaked Shivatzki ${ }^{1}$, Inbal Weiss ${ }^{1,3}$, Lilach M Friedman ${ }^{1}$, Noam Shomron ${ }^{2}$ and Karen B Avraham ${ }^{1 *}$

\begin{abstract}
Background: The mammalian inner ear contains sensory organs, the organ of Corti in the cochlea and cristae and maculae in the vestibule, with each comprised of patterned sensory epithelia that are responsible for hearing and balance. The development, cell fate, patterning, and innervation of both the sensory and nonsensory regions of the inner ear are governed by tight regulation involving, among others, transcription factors and microRNAs (miRNAs). In humans, mutations in specific miRNA genes are associated with hearing loss. In mice, experimental reduction or mutations of miRNAs in the inner ear leads to severe developmental and structural abnormalities. A comprehensive identification of miRNAs in the sensory epithelia and their gene targets will enable pathways of auditory and vestibular function to be defined.
\end{abstract}

Results: In this study, we used Next-Generation Sequencing (NGS) to identify the most prominent miRNAs in the inner ear and to define miRNA-target pairs that form pathways crucial for the function of the sensory epithelial cells. NGS of RNA from inner ear sensory epithelial cells led to the identification of 455 miRNAs in both cochlear and vestibular sensory epithelium, with 30 and 44 miRNAs found in only cochlea or vestibule, respectively. miR-6715-3p and miR-6715-5p were defined for the first time in the inner ear. Gene targets were identified for each of these miRNAs, including Arhgap12, a GTPase activating protein, for miR-6715-3p, implicating this miRNA in sensory hair cell bundle development, actin reorganization, cell adhesion and inner ear morphogenesis.

Conclusions: This study provides a comprehensive atlas of miRNAs in the inner ear sensory epithelia. The results provide further support of the essential regulatory role of miRNAs in inner ear sensory epithelia and in regulating pathways that define development and growth of these cells.

Keywords: Deafness, Inner ear, Sensory epithelia, RNA-seq, MicroRNAs

\section{Background}

miRNAs play an essential role in inner ear development [1]. miRNAs are small non-coding RNAs that regulate gene expression post-transcriptionally. By binding to sequences in the 3' untranslated region (UTR) of mRNAs, a miRNA can inhibit target mRNAs by translational suppression and mRNA destabilization [2]. miRNAs have been implicated in hearing loss in humans, since mutations in the

\footnotetext{
* Correspondence: karena@post.tau.ac.il

${ }^{\dagger}$ Equal contributors

'Department of Human Molecular Genetics and Biochemistry, Sackler Faculty of Medicine and Sagol School of Neuroscience, Tel Aviv University, Tel Aviv 69978, Israel

Full list of author information is available at the end of the article
}

seed region of miR-96 or its gene are associated with hearing loss in three extended families [3,4]. miRNAs were also reported in other human ear pathologies, including an elevation of miR-21 in cholesteatomas [5] and in vestibular schwannomas [6]. miRNAs were found to regulate the otitis media inflammatory response [7]. Their study in humans, however, has been hampered by the unavailability of inner ear RNA from human subjects, making the mouse an invaluable model for studying miRNA development and regulation in the inner ear [8]. In an ENUinduced mutant mouse exhibiting deafness and vestibular dysfunction, a mutation in the miR-96 seed region was found to be responsible for hearing loss, presumably due 
to the alteration in expression and hence function of both direct and indirect gene targets [9]. A number of mouse mutants affecting miRNA regulation through Dicer have been created, leading to developmental inner ear defects $[10,11]$. These conditional knock-out mouse mutants have been instrumental in demonstrating that miRNAs are vital for inner ear morphogenesis and development of the sensory epithelia and sensory neurons.

Expression of over 300 miRNAs have been reported in mouse and rat inner ears thus far, identified by microarray analysis $[8,10,12,13]$, with the majority of work performed on the triad mir-96, -182 , and -183 [14]. In order to further identify and characterize miRNAs in the mammalian inner ear, we used NGS for the first time to identify miRNAs in cochlear and vestibular sensory epithelia. Using this method, we identified over 500 inner ear miRNAs, both in common between and unique to each tissue. We further validated and characterized the expression of two miRNAs, miR-6715-3p and miR-6715$5 \mathrm{p}$, in the mouse inner ear. Vezatin, an adherens junctions transmembrane protein previously implicated in deafness [15] and Arhgap12, a Rho GTPase activating protein [16], were verified to be gene targets of miR6715-3p. Protocadherin 19, a member of the $\delta 2$ subclass of nonclustered protocadherins and associated with epilepsy and mental retardation [17], is a gene target of miR-6715-5p. Arhgap12, newly described in the inner ear, is expressed in the epithelial cell-cell junctions of the hair and supporting cells. The miR-6715-3p-Arhgap12 miRNA target pair defines a new regulatory pathway. Understanding these interactions may shed light on known and novel miRNAs, their effect on the development of normal and impaired hearing, and the mechanisms leading towards deafness.

\section{Results}

\section{RNA-seq derived miRNA transcription profile of the} mouse inner ear sensory epithelium

In order to identify miRNAs in the auditory and vestibular sensory epithelium, we sequenced short RNA molecules from cochlear and vestibular sensory epithelia by high-throughput RNA sequencing (RNA-seq). For the cochlea, the sensory epithelium consisted of the organ of Corti, including hair cells, supporting cells, and cells of the greater and lesser epithelial ridges, as well as mesenchymal and neuronal cells. For the vestibule, the sensory epithelium was derived from the saccule, utricle and the lateral and anterior cristae and included hair cells and supporting cells. The advantage of using high-throughput sequencing is that this technique allows for assessment of transcript expression in a given sample at a large dynamic range, from minute quantities to highly abundant ones, and does not require a-priori knowledge for the identification of modified and novel RNA molecules in addition to known ones. cDNA libraries from small RNAs were prepared from dissected sensory epithelium of post-natal day (P)0 mice. Subsequent analysis of sequence reads was done using miRNAkey, a tool for miRNA differential expression analysis [18]. Processed reads were aligned to the mature Mus musculus miRNA database (http://www.mir base.org), allowing one mismatch to occur between a read and the reference, due to isomiRs [19]. Sixty-six and $61.4 \%$ of the total reads from cochlear and vestibular sensory epithelia, respectively, were aligned to known mouse miRNAs, indicating a successful isolation of miRNAs. The majority of mapped reads in cochlear and vestibular sensory epithelia, $96.9 \%$ in the cochlea and $97.6 \%$ in the vestibule, were uniquely mapped to a single miRNA. Overall, 485 and 499 known miRNAs had an RPM (reads per million) mapping higher than 1 in the cochlear and vestibular sensory epithelium, respectively (Additional file 1: Table S1). These lists include all miRNAs that have been studied thus far in the mouse inner ear sensory epithelia, as well as miRNAs that have not been previously detected. Nine miRNAs were most highly expressed in the cochlea and the vestibule, with a similar order in both, except for miR-204-5p, which was the fourth most prevalent in the cochlea and the lowest in the vestibule (Table 1).

Differential expression among the known miRNAs was inferred by miRNAkey, based on the widely-accepted assumption that the number of reads mapped to each miRNA is relative to its level of expression [20,21]. The reads numbers in each sample were normalized to RPM values, and the relative expression levels of the different miRNAs in the two samples were calculated. In general, miRNA expression was similar in the cochlear and vestibular sensory epithelia. The majority of miRNAs with $\mathrm{RPM}>1$ were found in both tissues (455, or $86 \%$ ), with 30 found only in the cochlea and 44 found only in the vestibule (Figure 1A, Additional file 1: Table S1). The rates of the highest expressing miRNAs in each tissue were also highly similar between tissues (Pearson coefficient $=0.99 ; \mathrm{P}<10^{-8}$ ) (Figure $\left.1 \mathrm{~B}\right)$. We defined a miRNA as differentially expressed if the fold change between samples was higher than 4 and $\mathrm{P}<10^{-4}$ (Bonferroni corrected). Eleven miRNAs were differentially expressed in cochlear and vestibular sensory epithelia (Table 2).

\section{Targets for most highly expressed miRNAs}

miRNAs and targets exert their function through regulation of biological processes. As each miRNA can have multiple targets, we performed an analysis to discover additional mRNA genes that are computationally predicted to be targets of the three most highly expressed miRNAs, miR-182, miR-181a and miR-26a. Among numerous predictions, a few were of particular interest due to their involvement in auditory function. The targets were defined according to their presence in the list 
Table 1 Most highly expressed miRNAs in cochlear and vestibular sensory epithelium

\begin{tabular}{lccccc}
\hline miRNA name & preMir & Read count in cochlear sample & Cochlea RPM & Read count in vestibular sample & Vestibule RPM \\
\hline mmu-miR-182-5p & mmu-mir-182 & 2421025 & 453713.969 & 2746404 & 485527.023 \\
mmu-miR-181a-5p & mmu-mir-181a & 809893 & 151778.593 & 661755 & 116989.320 \\
mmu-miR-26a-5p & mmu-mir-26a & 254545 & 47703.193 & 243220 & 42998.001 \\
mmu-miR-204-5p & mmu-mir-204 & 129332 & 24237.559 & 84716 & 14976.641 \\
mmu-miR-27b-3p & mmu-mir-27b & 110796 & 20763.806 & 141616 & 25035.790 \\
mmu-let-7f-5p & mmu-let-7f & 86534 & 16216.968 & 103956 & 18378.013 \\
mmu-miR-127-3p & mmu-mir-127 & 85466 & 16016.819 & 131667 & 23276.942 \\
mmu-miR-22-3p & mmu-mir-22 & 80333 & 15054.865 & 92772 & 16400.833 \\
mmu-miR-183-5p & mmu-mir-183 & 79665 & 14929.678 & 94421 & 16692.354 \\
\hline
\end{tabular}

of genes that are associated with deafness in the Mouse Genome Informatics (MGI) (http://www.informatics. jax.org/diseasePortal/) database and predicted by two prediction algorithms, TargetScanMouse and miRanda (Table 3). All miRNA targets presented are involved in auditory function and hearing loss. As a few of the predicted targets overlap, we postulated that there might be underlying regulatory networks prompted by these miRNAs. This was further illustrated using gene ontology (GO) of biological-based processes, performed by geneMANIA (http://www.genemania.org), where 6/16 genes fell in the categories 'sensory perception of sound' and 'sensory perception of mechanical stimulus,' while 5/16 genes fell in the categories 'inner ear development' and 'ear development' (Additional file 2: Table S2). These predictions may set the basis for signaling pathways in the inner ear, subject to experimental validation.

\section{Identification of inner ear miRNAs}

In order to identify inner ear miRNAs, the genes associated with "impaired hearing" (MP:0006325) and "deafness" (MP:0001967) were downloaded from MGI (Additional file 3: Table S3). Since these genes are crucial for inner ear development and function, we predicted that miRNAs that reside in their introns may have a role in the inner ear. Out of the hearing associated genes, ten had pri-miRNAs

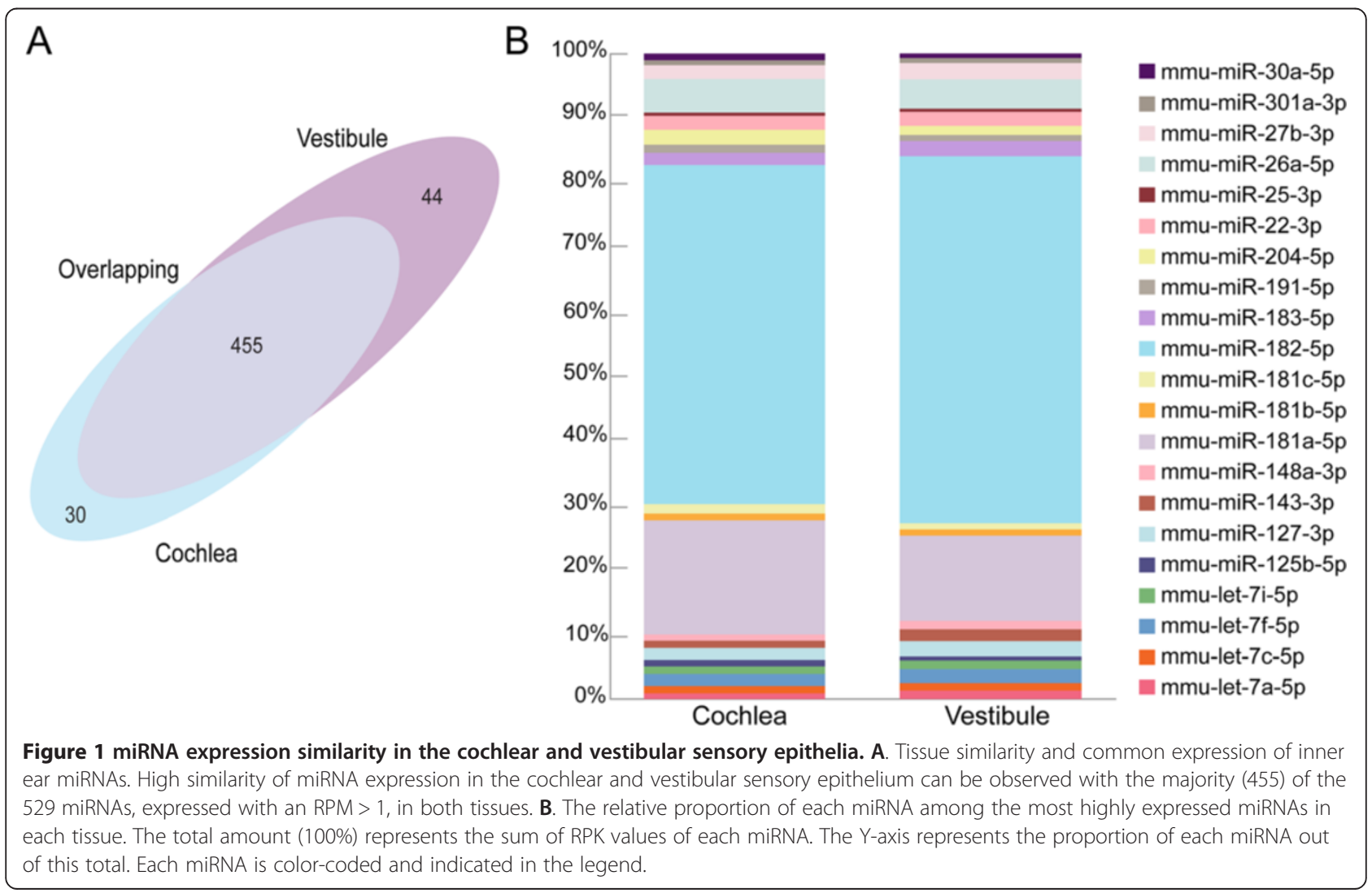


Table 2 Differentially expressed miRNAs in cochlear and vestibular sensory epithelium

\begin{tabular}{|c|c|c|c|c|c|c|c|c|c|}
\hline miRNA name & $\begin{array}{l}\text { Read } \\
\text { count } \\
\text { cochlea }\end{array}$ & $\begin{array}{c}\text { RPM } \\
\text { cochlea }\end{array}$ & $\begin{array}{c}\text { Read } \\
\text { count } \\
\text { vestibule }\end{array}$ & $\begin{array}{c}\text { RPM } \\
\text { vestibule }\end{array}$ & $\begin{array}{l}\text { Fold change } \\
\qquad(1>2)\end{array}$ & $\begin{array}{l}\text { Fold change } \\
\qquad(2>1)\end{array}$ & $\begin{array}{c}\text { Statistic } \\
(\text { Chi^^2 dist })\end{array}$ & P-value & $\begin{array}{c}\text { Corrected } \\
\text { P-value } \\
\text { (Bonferroni) }\end{array}$ \\
\hline mmu-miR-1298-5p & 156 & 29.235 & 3202 & 566.070 & 19.363 & 0.052 & 2731.789 & $0.00 E+00$ & $0.00 \mathrm{E}+00$ \\
\hline mmu-miR-375-3p & 665 & 124.625 & 8837 & 1562.262 & 12.536 & 0.080 & 6909.886 & $0.00 \mathrm{E}+00$ & $0.00 \mathrm{E}+00$ \\
\hline mmu-miR-211-5p & 234 & 43.853 & 2696 & 476.616 & 10.869 & 0.092 & 2026.071 & $0.00 E+00$ & $0.00 E+00$ \\
\hline mmu-miR-1298-3p & 16 & 2.998 & 116 & 20.507 & 6.839 & 0.146 & 73.212 & $0.00 E+00$ & $0.00 E+00$ \\
\hline mmu-miR-135b-5p & 216 & 40.480 & 1059 & 187.217 & 4.625 & 0.216 & 529.336 & $0.00 \mathrm{E}+00$ & $0.00 \mathrm{E}+00$ \\
\hline mmu-miR-10b-5p & 2584 & 484.256 & 12231 & 2162.275 & 4.465 & 0.224 & 5963.760 & $0.00 \mathrm{E}+00$ & $0.00 \mathrm{E}+00$ \\
\hline mmu-miR-6240 & 54 & 10.120 & 249 & 44.020 & 4.350 & 0.230 & 118.741 & $0.00 E+00$ & $0.00 E+00$ \\
\hline mmu-miR-3059-5p & 11 & 2.061 & 60 & 10.607 & 5.145 & 0.194 & 32.292 & 1.33E-08 & 1.17E-05 \\
\hline mmu-miR-153-3p & 933 & 174.850 & 175 & 30.938 & 0.177 & 5.652 & 541.720 & $0.00 E+00$ & $0.00 E+00$ \\
\hline mmu-miR-383-5p & 660 & 123.688 & 95 & 16.795 & 0.136 & 7.365 & 437.013 & $0.00 \mathrm{E}+00$ & $0.00 E+00$ \\
\hline mmu-miR-6715-5p & 78 & 14.618 & 12 & 2.121 & 0.145 & 6.890 & 50.139 & $1.43 \mathrm{E}-12$ & 1.27E-09 \\
\hline
\end{tabular}

in their introns (Additional file 4: Table S4). One primiRNA, expressed in both cochlear and vestibular sensory epithelia with an RPM $>1$, was found in the intron of deafness-related gene $\beta$-tectorin (Tectb) (Figure 2A), which is expressed in the mouse inner ear and associated with altered hearing [22]. This pri-miRNA produces two mature miRNAs, miR-6715-3p and miR-6715-5p (Figure 2B). These miRs were supported by 2.08 and 5.06 reads per million library reads, respectively (Figure $2 \mathrm{C}$ ). These miRs were also found in an analysis of sequences from mouse data sets derived from different tissues [23]. Provisional ID chr19_45771, available on miRBase (miR6715; MI0025026), was detected by an extremely low number of reads (0.0021-0.0126 per million library reads) in oocytes, embryonic day (E)7.5 embryo, E15.5 brain, hippocampus, heart, skin, muscle cells, lung and liver by RNA-seq.

\section{Expression of new inner ear miRNAs}

The expression of miR-6715a-3p and miR-6715a-5p, detected by RNA-seq, was validated by qRT-PCR analysis (Figure 3A). We found both miRNAs to be expressed in the sensory epithelium of the mouse cochlea, at E16, P0 and P8. The expression of both miRNAs was dynamic and increased with age, showing higher expression at P8 compared to P0 and E16, and at P0 compared to E16 $(\mathrm{P}<0.005)$. The two miRNAs were undetected in brain, liver, kidney and heart. This is consistent with

Table 3 Predicted mRNA targets for the most abundant miRNAs in the sensory epithelia of the inner ear

\begin{tabular}{ll}
\hline miRNA & Targets \\
\hline miR-182 & Bdnf, Ednrb, Rdx, Rere, Sox2 \\
miR-181a & Chd7, Grid1, Psap, Slc19a2, Tnfrsf11b \\
miR-26a & Atf2, Fbxo11, Gabrb3, Kcnq4, Rb1, Rere, Slc12a2, Slc19a2 \\
\hline
\end{tabular}

the previous RNA-seq detection [23]. The inner ear expression, and the absence or very low expression of the miRNAs in other tested tissues, suggests that they are inner ear-specific miRNAs. The expression prior to the onset of hearing suggests a developmental role for these miRNAs.

In order to explore the spatial expression pattern of the two inner ear miRNAs, in situ hybridization analysis was performed on inner ears derived from P0 C57/BL6 mice, at the same age the RNA-seq was performed. Consistent with the qRT-PCR results, expression of the two miRNAs was observed in P0 mice (Figure 3B). A clear staining of both miRNAs was observed in many parts of the inner ear, in spiral and vestibular ganglia, basilar membrane and Reissner's membrane, the stria vascularis and spiral ligament. In a higher magnification, in the cochlea, expression of both miRNAs was observed in the organ of Corti, in the spiral limbus and in particular in the hair and supporting cells. In the vestibular system, staining was observed in sensory epithelia of the saccule and the utricle, in hair and supporting cells.

\section{Target pathways in auditory and vestibular function}

In order to determine what pathway might be impacted by miR-6715-3p and miR-6715-5p regulation, we searched for potential targets. Targets were predicted by TargetScan Custom, which searches for a complementary 3'UTR given a putative seed sequence. miR-6715-3p and miR6715-5p were each predicted to target 201 and 206 targets, respectively, with ten overlapping targets (Additional file 5: Table S5). We examined which potential targets were inner ear-expressing genes, genes associated with hearing loss in human or mice, or genes encoding proteins involved in pathways relevant for inner ear function. These included protocadherin 19 (Pcdh19) [24] as a candidate target for 


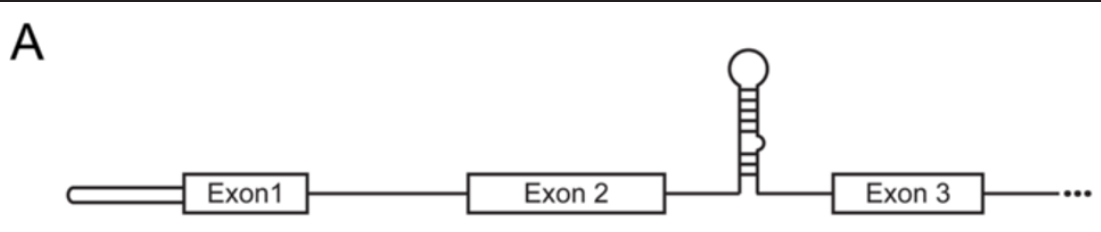

B
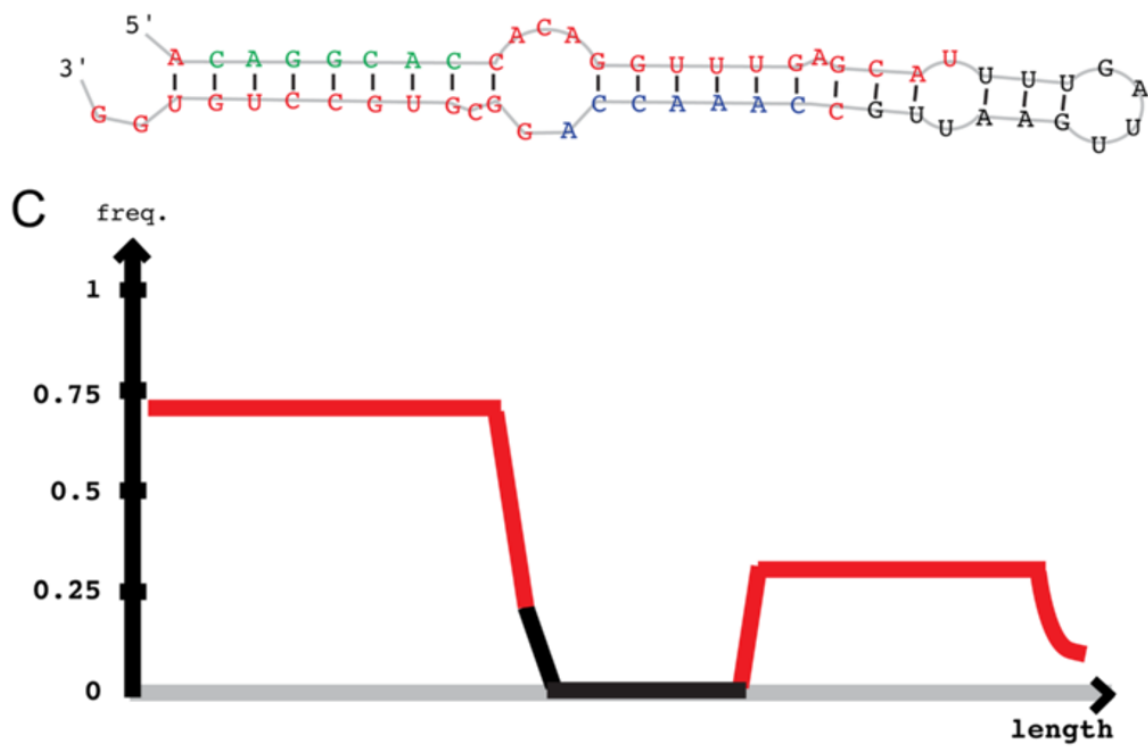

mmu-miR-6715-5p_MIMAT0029876

mmu-miR-6715-3p_MIMAT0029877

$5^{\prime}$ - acaggcaccacagguuugagcauuuugauugaauugccaaaccaggcgugccugugg - 3'

Figure 2 The genomic location, predicted structure and relative level of expression of miR-6715-3p and miR-6715-5p. A. pre-miR6715 is located in the second intron of the Tectb gene. B. miRDeep2 prediction of the RNA secondary hairpin structure of the unprocessed miR-6715-3p, miR-6715-5p, and loop. The seed region of miR-6715-3p is indicated in blue and of miR-6715-5p in green. C. Middle density plot shows the distribution of reads in the predicted precursor sequence, as produced by miRDeep2.

miR-6715-5p, and vezatin (Vezt) [15], Scn8a [25] and Arhgap12 [16] as candidate targets for miR-6715-3p.

To test whether these potential targets have a direct interaction with miR-6715-3p and miR-6715-5p, we examined luciferase activity by inserting the 3'UTR of the potential targets downstream of a luciferase gene. Transfections of these constructs, together with a pre miR-6715 expressing vector into HEK293T cells, and measurement of the relative luciferase activity compared to a mutant 3'UTR construct, were indicative of targeting. Pcdh19 was found to be a direct target of miR-6715a-5p (Figure 4A), with a reduction of luciferase activity by approximately $35 \%$. Vezt and Arhgap 12 were found to be direct targets of miR-6715-3p, with a reduction of luciferase activity by $55 \%$ and $15 \%$, respectively (Figure $4 \mathrm{~B}, \mathrm{C}$ ). Snc8a is not a direct target of miR-6715a-3p, as luciferase activity was not reduced (Figure 4D).

Arhgap12 was chosen for further analysis, due to its putative role as a GTPase activating protein (GAPs) for small G proteins and expression in epithelial cell-cell junctions [16]. Temporal expression of Arhgap12 mRNA was evaluated by qRT-PCR in the cochlear sensory epithelium. In the cochlea, Arhgap12 was expressed at E16, P0 and P8, with a small, though not significant, increase in age (Figure 5). This protein was not previously characterized in the inner ear. We found Arhgap12 expression, using a commercial antibody examined for specificity by peptide competition (Additional file 6) in the organ of Corti, in hair and supporting cells, the stria vascularis, spiral ligament and Reissner's membrane (Figure 6A, B). The strongest expression was observed in nerve cells, in the spiral ganglia (Figure $6 \mathrm{C}$ ). In the vestibule, expression was found in the hair and supporting cells (Figure 6D). In whole mount preparations, Arhgap12 was found to be present in hair and supporting cells (Figure 7A-C). Its expression was observed in the same hair cell and cuticular plate planes as ZO-1, but did not overlap (Figure 7A-C). The outer hair cell and 


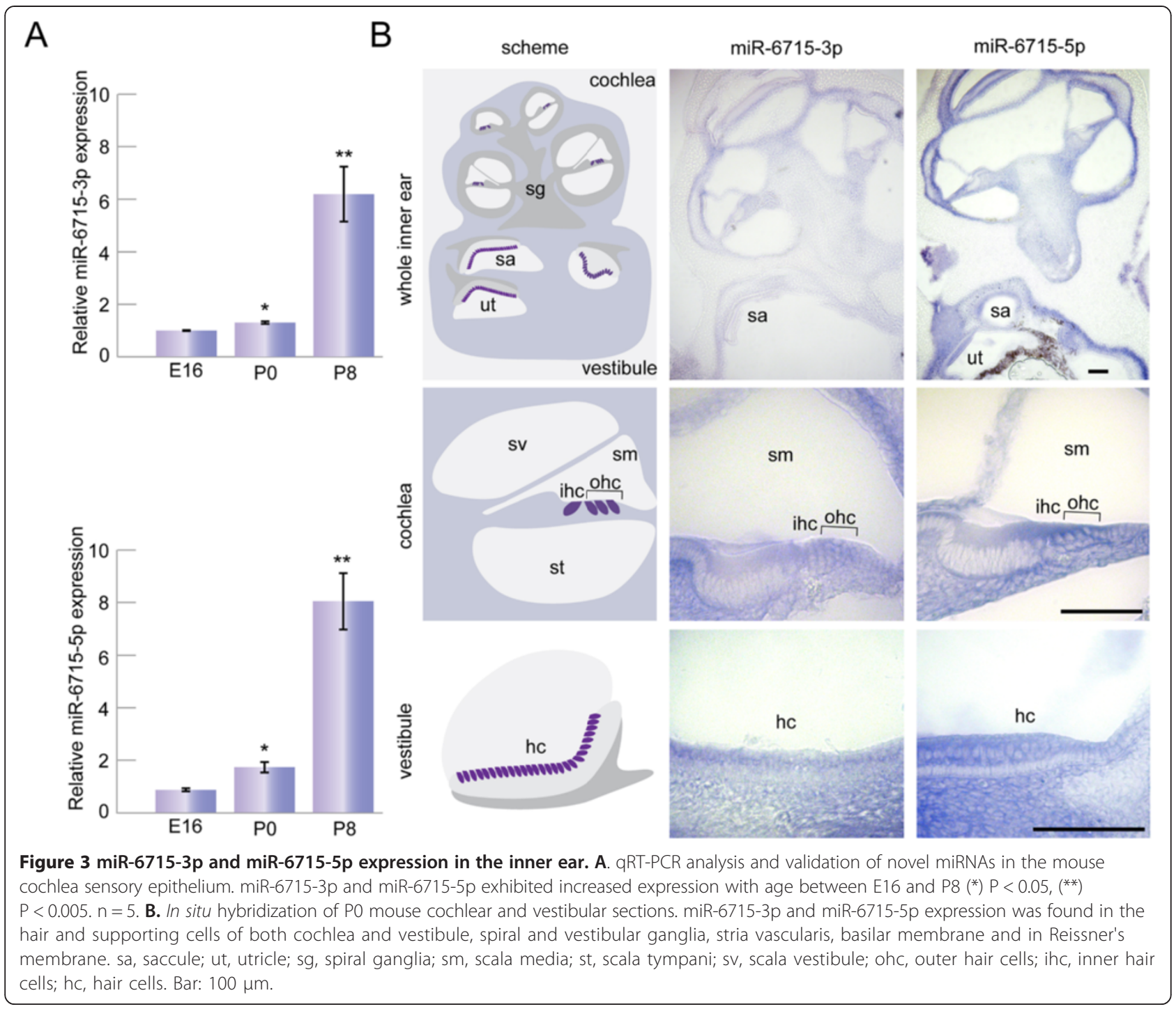

Deiter cell junctions expresses ZO-1, while Arhgap12 appears absent in these junctions. Arhgap12 appears in the kinocilium (Figure 6B).

\section{Discussion}

The function of miRNAs and their targets in the mammalian inner ear are now being discovered, as the link between miRNAs and hearing and deafness has been established. The inner ear is a complex tissue with multiple cell types, with miRNAs involved in fine-tuning the multitude pathways required for functional hearing. While there are a relatively small number of miRNAs in mammals, their role in regulation far surpasses their number, since each miRNA can exert its effect on hundreds of downstream targets [26]. Hundreds of miRNAs have been discovered in the inner ear; only a subset of these miRNAs have validated targets (reviewed in Table 1 [27]).
Our study was designed to profile the general expression of miRNAs in the inner ear and a comparison of the auditory and vestibular epithelia. We chose the time point of p0 since work by our group $[10,13]$ and others have demonstrated that miRNAs are expressed and functional at this stage, including miRNA-96, which is essential for hearing $[3,9]$. RNA-seq analysis led to the identification of over 500 miRNAs, with miR-182 as the most highly expressed miRNA in both sensory epithelia (Table 1) and accounting for more than $50 \%$ of the 20 most highly expressed miRNAs. A few targets have been confirmed for miR-182, including Sox2, Clic5 and Tbx1 [28-30]. The second most abundant miRNA in the cochlear and vestibular sensory epithelia, miR-181a-5p, has no known validated targets in the inner ear. This miRNA has been studied in other systems and found to have a proproliferative role in cultured human myeloid leukemia cells [31] and regulates thymic selection in mouse T-cells 

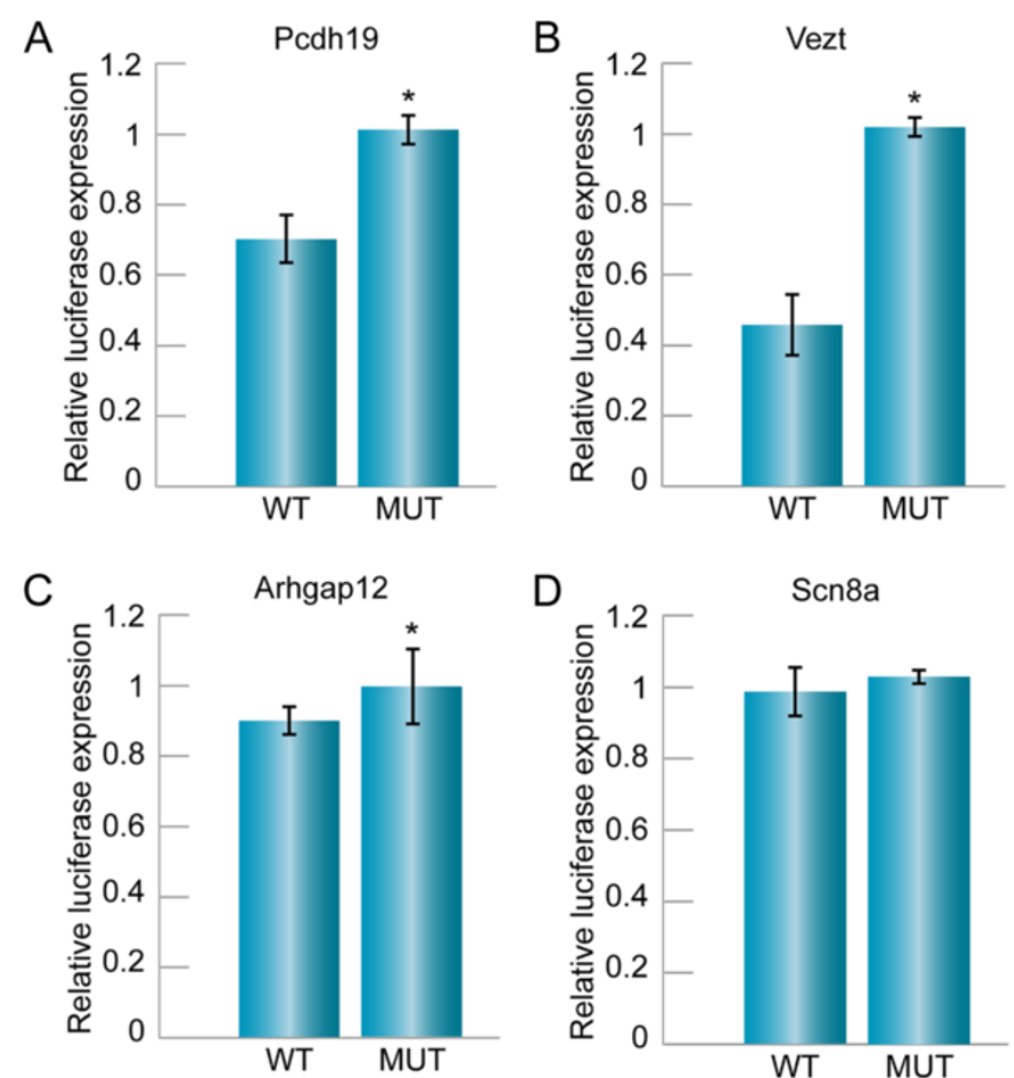

Figure 4 miRNA-target prediction validation and expression. Using TargetScan, potential targets of miR- 6715-3p and miR-6715-5p were identified, based on the complimentarily of the seed region and 6-7 nt sequence of the target mRNA. To test the possibility of miR-target regulation, luciferase assays were performed. A. Protocadherin 19 as a potential target of miR-6715-5p. Wild-type $3^{\prime} U$ TR had approximately $65 \%$ of luciferase activity as compared to the mutant. $\left(^{*}\right) \mathrm{P}<0.05$. B. Vezatin as a potential target of miR-6715-3p. Wild-type $3^{\prime} U T R$ had approximately $45 \%$ of luciferase activity as compared to the mutant (*) $P<0.05$. C. Activator for the Rho-type GTPases Arhgap12 as a potential target of miR- 6715-3p. Wild-type $3^{\prime} U T R$ had approximately $85 \%$ of luciferase activity as compared to the mutant. $\left(^{*}\right) \mathrm{P}<0.05$. D. Scn8a is not a direct target for miR-6715-3p, as the luciferase activity was not reduced.

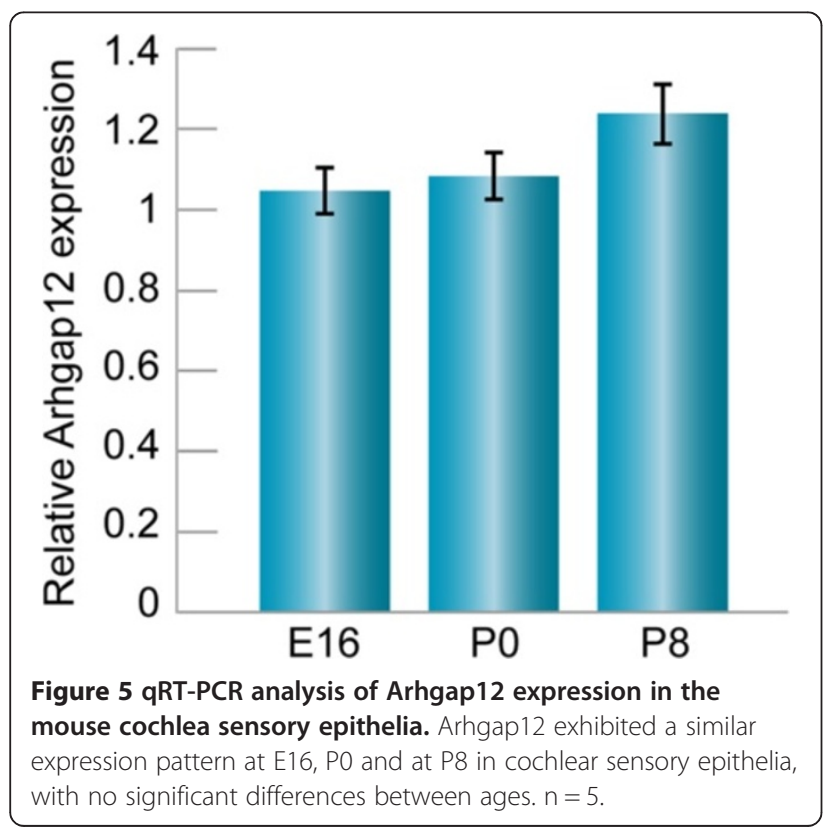

[32]. The role of this miRNA has been studied in the avian inner ear, where it has been shown to promote proliferation [33].

We validated a number of targets for miR-6715-3p and miR-6715-5p. Predicted targets for the miRNAs described in this study have direct or potential implications for hearing loss. Pcdh19 is expressed in the zebrafish inner ear [24]. Mutations in protocadherin 19 (PCDH19) have been associated with $\mathrm{X}$ chromosome-linked epilepsy and mental retardation and it is a member of the protocadherin family of proteins [17]. This family includes protocadherin 15 (Pcdh15), with a known function in the tip links of the stereocilia of the inner ear in conjunction with cadherin 23 [34] and has been associated with multiple mutations in both non-syndromic and syndromic hearing loss in humans $[35,36]$.

Vezatin (Vezt) is an adherens junctions transmembrane protein, and while it is ubiquitously expressed in these junctions, it is especially prevalent in in hair and supporting cells of the mouse inner ear [15]. Vezt mutant mice 

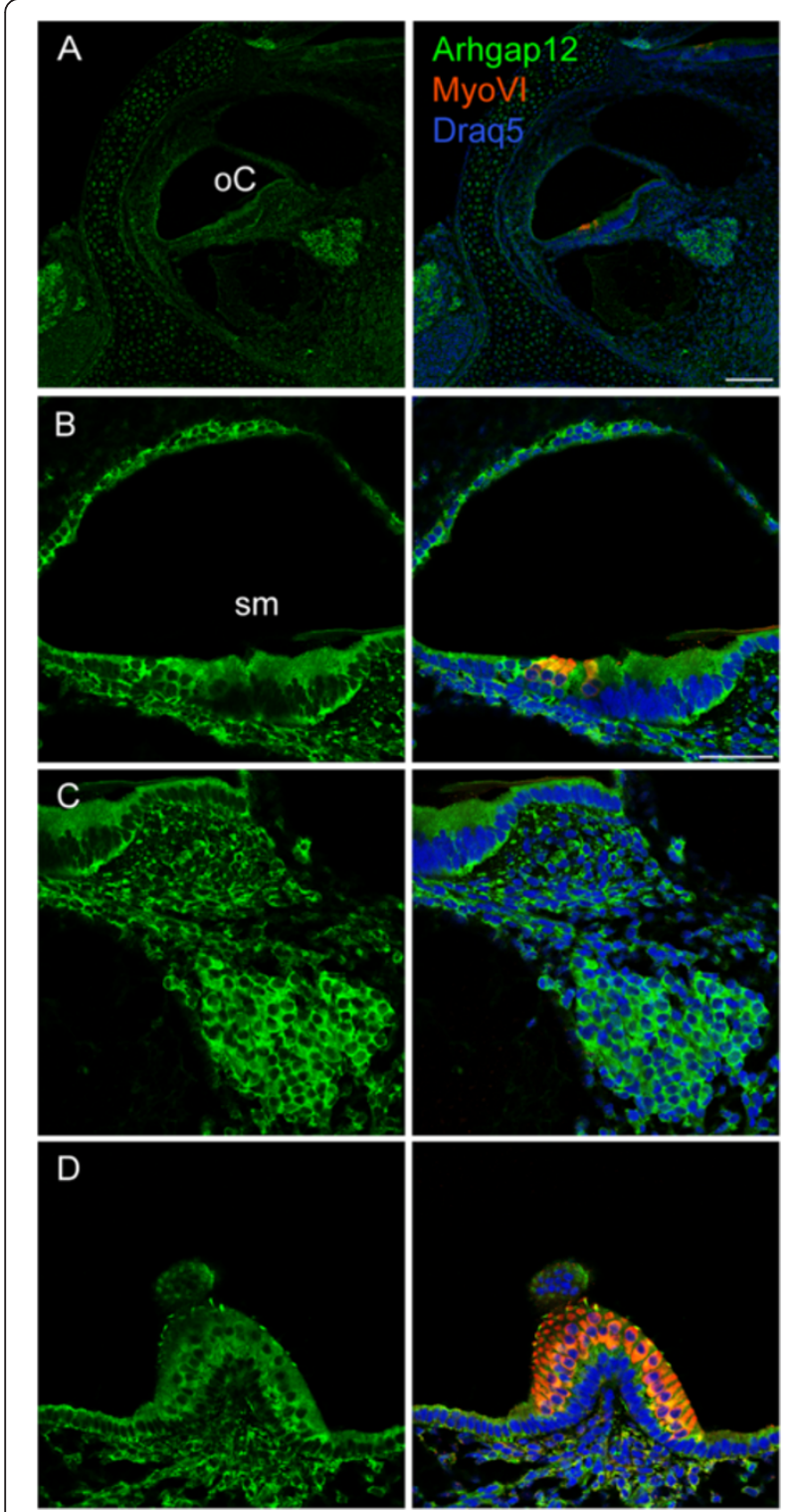

Figure 6 Arhgap12 protein expression in mouse inner ear sections. Immunohistochemistry of Arhgap12 (green) in P0 inner ear sections, with hair cells stained by myosin VI (red) and nuclei stained by Draq5 (blue). A. Arhgap12 was detected in the organ of Corti, in hair and supporting cells, stria vascularis, spiral ligament and Reissner's membrane. B. Magnification of the organ of Corti, with localization of Arhgap12 in epithelial cells. C. Arhgap12 is localized to the spiral ganglia. D. In the vestibular system, Arhgap12 is present in hair and supporting cells in the crista. Bar: A, $100 \mu \mathrm{m}$; B-D, $50 \mu \mathrm{m}$.

suffer from late onset progressive hearing loss, due to hair cell apoptosis. Most compelling, the resistance of the organ of Corti to mechanical stress was reduced when vezatin was absent in the inner ears of conditional mutants. These studies led to the prediction that vezation is required to maintain junction integrity and is crucial to protect the junctions from mechanical stress due to sound trauma.
Arhgap12, a Rho GTPase activating protein (GAP) family member, is a component of cell-cell junctions in kidney epithelial cells [16]. While not previously known to be expressed in the inner ear, its target, Rac1, localizes to hair and supporting cells, specifically in the stereocilia and kinocilium of the hair cells. It was found to be involved in hair cell morphogenesis, specifically in hair bundle development, epithelia actin assembly and cell adhesion. Inactivation of Rac1 specifically in the otic epithelium leads to defects in cochlear morphogenesis, hair bundle orientation and cellular organization of the organ of Corti [37]. Arhgap12 was found to target Rac1 and inactivates it by increasing GTP hydrolysis [38]. Here we suggest a key role for miR-6715-3p in targeting Arhagap12 and in this fashion, influencing Rac1 in the inner ear (Figure 8).

Arhgap12, a GAP protein, is involved in controlling the time period in which Rac1 will remain active. In MDCK cells, Arhgap12 expression overlaps with Rac1 expression [38,39], probably due to the regulation of Arhgap12 on Rac1 at this time point. In the inner ear, besides the actin-rich areas, Rac1 is localized to hair cells and in particular, the kinocilium [37]. In accordance with our data, Arhgap12, at P0, with expression in the hair cells and kinocilium, might be regulating by Rac1 at this time, when the hair bundle is still developing and needs to be under close regulation. The kinocilium is necessary for proper development [40] and may have a structural role in guiding the architecture of the hair bundle and/or be involved in G-protein signaling in the kinocilium. If the latter is true, then Arhgap12 may regulate its GTPase activity. In our model of the miR-6715-3p circuit in the inner ear, we propose that the miRNA indirectly enhances the action of Rac1 and therefore may promote hair bundle development, actin reorganization, cell adhesion and inner ear morphogenesis.

\section{Conclusions}

The identification of inner ear-related miRNAs by RNAseq analysis demonstrates that the dataset is reliable not only for characterizing expression profiles of known miRNAs, but also for discovery of novel miRNAs in the inner ear. Further investigation of these miRNAs may shed light on their regulatory roles in various molecular pathways underlying the development of the embryonic inner ear.

\section{Methods}

\section{RNA isolation}

All procedures involving animals were approved and met the guidelines described in the National Institutes of Health Guide for the Use of Laboratory Animals and 

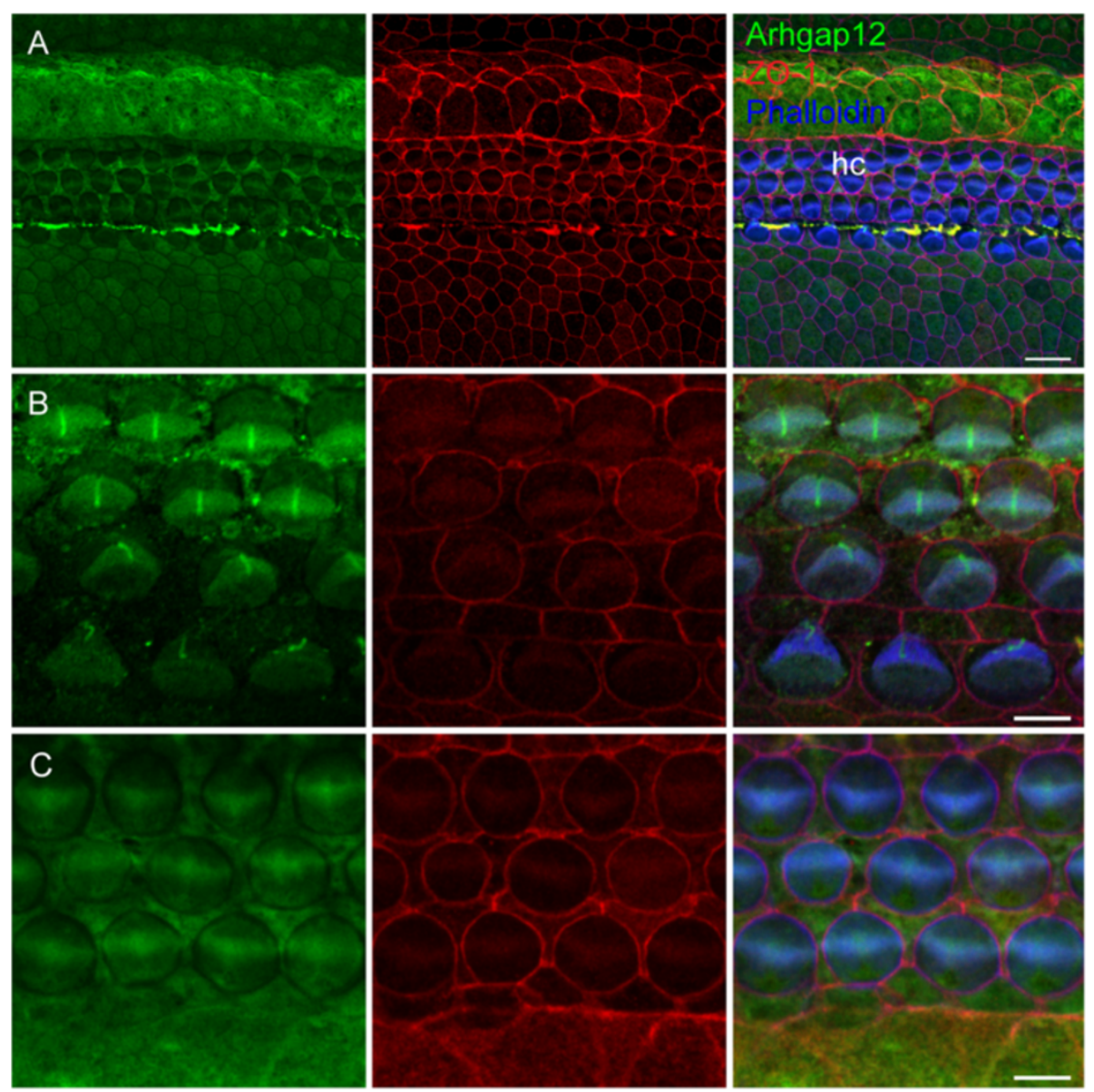

Figure 7 Arhgap12 protein expression in mouse inner ear whole-mount preparations. Immunohistochemistry of Arhgap12 (green) in whole mount preparations of PO mouse cochleas, with cell junctions stained by ZO-1 (red) and actin stained by phalloidin (blue). A. Arhgap12 is localized to the hair and supporting cells of the sensory epithelium. B. In a higher magnification of A, Arhgap12 is localized to the hair bundle and the kinocilium. C. Additional plane showing mutual exclusion of localization of Arhagp12 and ZO-1. hc, hair cells. Bar: A, $10 \mu \mathrm{m} ; \mathbf{B}, 5 \mu \mathrm{m}$.

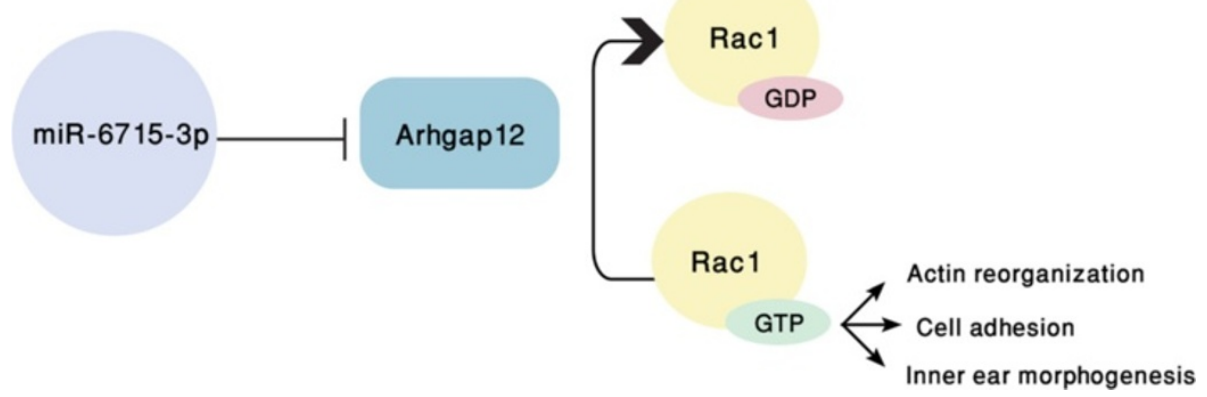

Figure 8 A newly identified miRNA-target regulatory pathway in the inner ear. A suggested pathway for miR-6715-3p and Arhgap12, whereby miR-6715-3p down-regulates expression of Arhgap12, which in turn blocks the hydrolysis of Rac1 (Ras-Related C3 Botulinum Toxin Substrate 1) GTPase. This may result in increased cell adhesion, actin reorganization and inner ear morphogenesis. 
approved by the Animal Care and Use Committee of Tel Aviv University (M-10-087). Cochlear and vestibular sensory epithelia were dissected from 12 inner ears of 6 P0 C57Bl/6 J mice. The cochlear sensory epithelia included the organ of Corti with some attached membranes, while the vestibular sensory epithelia included the saccule, utricle, lateral crista and anterior crista. The dissected sensory epithelia were placed immediately in Qiazol lysis buffer (Qiagen) on ice and stored at $-80^{\circ} \mathrm{C}$ for several hours. The sensory epithelia was then thawed and total RNA was isolated, using the miRNeasy Mini Kit (Qiagen) without DNAse, which enables purification of total RNA of $>18 \mathrm{nt}$. The isolated RNAs from the samples were precipitated in sodium acetate and glycogen in $80 \%$ ethanol (Ambion protocol), in order to further clean and concentrate the RNA samples. The precipitated RNA samples were dissolved in nuclease-free water and their quality was assessed by $1.2 \%$ agarose gel $(90 \mathrm{~V})$.

\section{RNA-sequencing}

Twelve cochlear epithelia were pooled and twelve vestibular epithelia were pooled, and each pool was used to create a library for MPS and sequenced. No biological repeats were performed. miRNA libraries for MPS were created using the TruSeq SmallRNA SamplePrep Kit (Illumina), with one modification. Instead of running the samples on acrylamide gel in order to isolate the miRNA band, the samples were loaded on a $4 \%$ agarose E-Gel and the purification was performed with the Qiagen Kit. The libraries were multiplexed and $2.5 \mathrm{pM}$ of each library was sequenced at SR $\times 36$ bp using the Illumina Genome Analyzer IIx in the same lane at the Functional Genomics Laboratory at Tel Aviv University.

\section{miRNA sequence analysis}

A total of $8,763,589$ and $10,048,818$ raw sequences of small RNAs were found in the cochlear and vestibular samples, respectively. Small RNA adapter sequences were clipped using fastq-mcf (ea-utils: http://code.google.com/ p/ea-utils/), discarding sequences shorter than 16 nucleotides and adapter dimers. Following adapter clipping, 8,340,741 and 9,441,679 reads remained. Differential expression of the resulting sequences was performed using miRNAkey. Specifically, sequence alignment was performed using Burrows-Wheeler Aligner (BWA) [41] against mouse mature miRNA sequences (downloaded from miRBase (Release 20: June 2013; http://www.mirbase.org/), while allowing one mismatch between read and reference. The read count for each read was normalized using the RPM method. Differential expression was tested using chi-squared proportion testing and corrected using the Bonferroni correction for multiple comparisons. Novel miRNA prediction was performed using miRDeep2 [42].

\section{qRT-PCR}

Cochlear sensory epithelia of E16, P0 and P8 C57BL/6 mice was dissected. Small RNAs were extracted using the miRNeasy Mini Kit (QIAGEN). Custom-made probes, designed by Applied Biosystems, were used to detect miRNAs. miRNAs and U6B RNA (endogenous control) were reverse transcribed using the High Capacity cDNA Reverse Transcription Kit (Applied Biosystems). The qRTPCR reaction was conducted using the FastStart Universal Probe Master (Roche) in the StepOne Plus qRT-PCR machine (Applied Biosystems). All miRNAs expression was normalized to the expression of U6B. At each age, sensory epithelia from 4-5 mice were pooled and five RT-PCR experiments were performed.

\section{In situ hybridization}

Inner ears of P0 C57BL/6 mice were dissected and fixed with $4 \%$ paraformaldehyde. Whole mount in situ hybridization analysis was performed using the Exiqon protocol. miRCURY LNA ${ }^{\mathrm{mi}}$ microRNA detection custommade probes, labeled with digoxygenin (DIG) (Exiqon), were used for the detection of novel miRNAs. Probes were hybridized with the tissue, $20-22^{\circ} \mathrm{C}$ below $\mathrm{Tm}$ of the probe. Probes were detected with the anti-DIG-AP (alkaline phosphatase conjugated) antibody (Roche), and the color reaction was developed using the NTB/BCIP (Sigma). The ears were frozen and cryosectioned to 10$18 \mu \mathrm{m}$ sections and mounted. Images were taken using the Zeiss Aviovert200 M microscope. Three experiments were performed, and 3-5 ears were included in each experiment.

\section{miRNA target prediction}

Target prediction for all miRNAs was predicted by TargetScan Custom (Release 5.2; http://www.targetscan.org/ vert_50/seedmatch.html), TargetScanMouse (Release 5.2, http://www.targetscan.org/mmu_50/) and/or miRanda (http://www.microrna.org/microrna/home.do). Chosen potential targets were compared to gene expression databases (SHIELD; https://shield.hms.harvard.edu/) and known hearing loss genes and loci (Hereditary Hearing Loss Homepage, http://hereditaryhearingloss.org).

\section{Luciferase assay}

3' UTRs of chosen potential targets were cloned into the pGL3 luciferase reporter vector, downstream to the luciferase reporter gene, creating the 3'UTR-wt-pGL3 vector. The first three nucleotides of the miRNA binding site in the 3'UTR were mutated by site directed mutagenesis, creating the 3'UTR-mut-pGL3 vector. The miR-6715 miRNA expression vector was created by cloning the genomic polycistronic miR-6715-3p and miR-6715-5p into the miRvec expression vector (obtained as a gift from Reuven Agami) [43]. HEK293T cells were transiently 
transfected with either 3'UTR-wt-pGL3 or with 3'UTRmut-pGL3, for each of the tested targets, together with the corresponding miRNA expression vector and Renilla expressing vector. The luciferase reporter assay was performed 48 hours following transfection using the Dual-Luciferase ${ }^{\bullet}$ Reporter assay system (Promega). Three experiments were conducted, with duplicates.

\section{Immunohistochemistry}

Whole mount and paraffin sections of P0 C67/BL6 mouse inner ear were prepared for staining as previously described [44]. Myosin VI was used to stain hair cells, phalloidin to stain actin and Draq5 to stain nuclei. The following antibodies were used: goat-anti-Arhgap12 antibody (Santa Cruz), 1:100; mouse-anti-ZO-1 (Zymed), 1:100; rabbit-anti-myosin VI (Proteus BioSciences) 1:200; and Draq5 1:600 (Abcam). Antibody specificity of Arhgap12 was assessed by a competition assay in HCT116 cells, with blocking by an Arhagap12 peptide (Santa Cruz) (Additional file 6).

\section{Statistics}

For qRT-PCR and luciferase assays, the Student's two-tailed $t$ test $\mathrm{P}$ values of less than 0.05 were considered to be statistically significant and those of less than 0.005 were considered to be highly statistically significant. The data in the figures are presented by mean + SEM. Differential expression of miRNAs between paired samples was measured using a chi-squared statistic. $P$-values are calculated for the null hypothesis of no differential expression between the two samples. Final $P$-values were corrected using the Bonferroni correction for multiple hypotheses testing [18].

\section{Availability of supporting data}

The RNA-seq data from this study is available in the NCBI Sequence Read Archive (SRA) (http://www.ncbi. nlm.nih.gov/sra), under accession number SRP043019.

\section{Additional files}

Additional file 1: Table S1. miRNAkey output table for cochlear vs. vestibular miRNA differential expression. miRNA differential expression analysis includes the read count, RPM, fold change and comparison statistics for each miRNA transcript.

Additional file 2: Table S2. Predicted targets categorized by gene ontology (GO) of biological-based processes.

Additional file 3: Table S3. Genes associated with "impaired hearing" and "deafness" impairment in MGl.

Additional file 4: Table S4. Expression of pri-miRNAs found inside introns of "impaired hearing" and "deafness" genes, as defined in MGI.

Additional file 5: Table S5. Predicted targets for miR-6715-3p and miR-6715-5p. Source: TargetScanHuman Custom Release 5.2: June 2011.

Additional file 6 . Specificity of the Arhgap12 antibody was tested by a peptide competition assay. HCT116 cells were stained with an Arhgap12 antibody and an Arhgap12 peptide. No staining of Arhgap12 was observed in the blocked cells. Bar: $25 \mu \mathrm{m}$.
Competing interests

The authors declare that they have no competing interests.

\section{Authors' contributions}

$A R, L M F, N S$ and KBA designed and coordinated the study. AR and SS collected the inner ear samples. AR, KU, SS, IW and LMF generated the experimental data. OI and LMF performed the computational analysis of the data. AR, OI, KU, NS and KBA contributed to the writing of the manuscript. All authors read and approved the final manuscript.

\section{Acknowledgements}

This study was supported by the Israel Science Foundation (grant no. 1320/11) National Institutes of Health (NIDCD) R01DC011835, I-CORE Gene Regulation in Complex Human Disease Center No. 41/11 and a fellowship from the Edmond J. Safra Center for Bioinformatics at Tel Aviv University. We thank Varda Oron-Karni for her aid with RNA-seq.

\section{Author details}

${ }^{1}$ Department of Human Molecular Genetics and Biochemistry, Sackler Faculty of Medicine and Sagol School of Neuroscience, Tel Aviv University, Tel Aviv 69978, Israel. ${ }^{2}$ Department of Cell and Developmental Biology, Sackler Faculty of Medicine and Sagol School of Neuroscience, Tel Aviv University, Tel Aviv 69978, Israel. ${ }^{3}$ Present address: Clalit Health Services, Migdal HaEmek Clinic, Tel Aviv, Israel.

Received: 9 March 2014 Accepted: 13 June 2014

Published: 18 June 2014

\section{References}

1. Li H, Fekete DM: MicroRNAs in hair cell development and deafness. Curr Opin Otolaryngol Head Neck Surg 2010, 18(5):459-465.

2. Guo H, Ingolia NT, Weissman JS, Bartel DP: Mammalian microRNAs predominantly act to decrease target mRNA levels. Nature 2010, 466(7308):835-840.

3. Mencia A, Modamio-Hoybjor S, Redshaw N, Morin M, Mayo-Merino F, Olavarrieta L, Aguirre LA, del Castillo I, Steel KP, Dalmay T, Moreno F, Moreno-Pelayo MA: Mutations in the seed region of human miR-96 are responsible for nonsyndromic progressive hearing loss. Nat Genet 2009, 41(5):609-613.

4. Solda G, Robusto M, Primignani P, Castorina P, Benzoni E, Cesarani A, Ambrosetti U, Asselta R, Duga S: A novel mutation within the MIR96 gene causes non-syndromic inherited hearing loss in an Italian family by altering pre-miRNA processing. Hum Mol Genet 2012, 21(3):577-585.

5. Friedland DR, Eernisse R, Erbe C, Gupta N, Cioffi JA: Cholesteatoma growth and proliferation: posttranscriptional regulation by microRNA-21. Otol Neurotol 2009, 30(7):998-1005.

6. Cioffi JA, Yue WY, Mendolia-Loffredo S, Hansen KR, Wackym PA, Hansen MR: MicroRNA-21 overexpression contributes to vestibular schwannoma cell proliferation and survival. Otol Neurotol 2010, 31(9):1455-1462.

7. Song JJ, Kwon SK, Cho CG, Park SW, Chae SW: Microarray analysis of microRNA expression in LPS induced inflammation of human middle ear epithelial cells (HMEECs). Int J Pediatr Otorhinolaryngol 2011, 75:648-651.

8. Weston MD, Pierce ML, Rocha-Sanchez S, Beisel KW, Soukup GA: MicroRNA gene expression in the mouse inner ear. Brain Res 2006, 1111(1):95-104.

9. Lewis MA, Quint E, Glazier AM, Fuchs H, De Angelis MH, Langford C, van Dongen S, Abreu-Goodger C, Piipari M, Redshaw N, Dalmay T, Moreno-Pelayo MA, Enright AJ, Steel KP: An ENU-induced mutation of miR-96 associated with progressive hearing loss in mice. Nat Genet 2009, 41(5):614-618.

10. Friedman LM, Dror AA, Mor E, Tenne T, Toren G, Satoh T, Biesemeier DJ, Shomron N, Fekete DM, Hornstein E, Avraham KB: MicroRNAs are essential for development and function of inner ear hair cells in vertebrates. Proc Natl Acad Sci USA 2009, 106(19):7915-7920.

11. Soukup GA, Fritzsch B, Pierce ML, Weston MD, Jahan I, McManus MT, Harfe BD: Residual microRNA expression dictates the extent of inner ear development in conditional Dicer knockout mice. Dev Biol 2009, 328(2):328-341.

12. Patel M, Cai Q, Ding D, Salvi R, Hu Z, Hu BH: The miR-183/Taok1 target pair is implicated in cochlear responses to acoustic trauma. PLoS One 2013, 8(3):e58471. 
13. Elkan-Miller T, Ulitsky I, Hertzano R, Rudnicki A, Dror AA, Lenz DR, Elkon R, Irmler M, Beckers J, Shamir R, Avraham KB: Integration of transcriptomics, proteomics, and microRNA analyses reveals novel microRNA regulation of targets in the mammalian inner ear. PLoS One 2011, 6(4):e18195.

14. Kuhn S, Johnson SL, Furness DN, Chen J, Ingham N, Hilton JM, Steffes G, Lewis MA, Zampini V, Hackney CM, Masetto S, Holley MC, Steel KP, Marcotti W: miR-96 regulates the progression of differentiation in mammalian cochlear inner and outer hair cells. Proc Natl Acad Sci U S A 2011, 108(6):2355-2360

15. Bahloul A, Simmler MC, Michel V, Leibovici M, Perfettini I, Roux I, Weil D, Nouaille S, Zuo J, Zadro C, Licastro D, Gasparini P, Avan P, Hardelin JP, Petit $C$ : Vezatin, an integral membrane protein of adherens junctions, is required for the sound resilience of cochlear hair cells. EMBO Mol Med 2009, 1(2):125-138

16. Matsuda M, Kobayashi Y, Masuda S, Adachi M, Watanabe T, Yamashita JK, Nishi E, Tsukita S, Furuse M: Identification of adherens junction-associated GTPase activating proteins by the fluorescence localization-based expression cloning. Exp Cell Res 2008, 314(5):939-949.

17. El-Amraoui A, Petit C: Cadherins as targets for genetic diseases. Cold Spring Harb Perspect Biol 2010, 2(1):a003095.

18. Ronen R, Gan I, Modai S, Sukacheov A, Dror G, Halperin E, Shomron N: miRNAkey: a software for microRNA deep sequencing analysis. Bioinformatics 2010, 26(20):2615-2616.

19. Zhou H, Arcila ML, Li Z, Lee EJ, Henzler C, Liu J, Rana TM, Kosik KS: Deep annotation of mouse iso-miR and iso-moR variation. Nucleic Acids Res 2012, 40(13):5864-5875.

20. Marioni JC, Mason CE, Mane SM, Stephens M, Gilad Y: RNA-seq: an assessment of technical reproducibility and comparison with gene expression arrays. Genome Res 2008, 18(9):1509-1517.

21. Mortazavi A, Williams BA, McCue K, Schaeffer L, Wold B: Mapping and quantifying mammalian transcriptomes by RNA-Seq. Nat Methods 2008 5(7):621-628.

22. Ghaffari R, Aranyosi AJ, Richardson GP, Freeman DM: Tectorial membrane travelling waves underlie abnormal hearing in Tectb mutant mice. Nat Commun 2010, 1:96.

23. Castellano L, Stebbing J: Deep sequencing of small RNAs identifies canonical and non-canonical miRNA and endogenous siRNAs in mammalian somatic tissues. Nucleic Acids Res 2013, 41(5):3339-3351.

24. Liu Q, Chen Y, Kubota F, Pan JJ, Murakami T: Expression of protocadherin19 in the nervous system of the embryonic zebrafish. Int J Dev Biol 2010, 54(5):905-911.

25. Mackenzie FE, Parker A, Parkinson NJ, Oliver PL, Brooker D, Underhill P, Lukashkina VA, Lukashkin AN, Holmes C, Brown SD: Analysis of the mouse mutant Cloth-ears shows a role for the voltage-gated sodium channel Scn8a in peripheral neural hearing loss. Genes Brain Behav 2009, 8(7):699-713.

26. Bartel DP: MicroRNAs: target recognition and regulatory functions. Cell 2009, 136(2):215-233.

27. Ushakov K, Rudnicki A, Avraham KB: MicroRNAs in sensorineural diseases of the ear. Front Mol Neurosci 2013, 6:52.

28. Weston MD, Pierce ML, Jensen-Smith HC, Fritzsch B, Rocha-Sanchez S, Beisel KW, Soukup GA: MicroRNA-183 family expression in hair cell development and requirement of microRNAs for hair cell maintenance and survival. Dev Dyn 2011, 240(4):808-819.

29. Gu C, Li X, Tan Q, Wang Z, Chen L, Liu Y: MiR-183 family regulates chloride intracellular channel 5 expression in inner ear hair cells. Toxicol In Vitro 2013, 27(1):486-491.

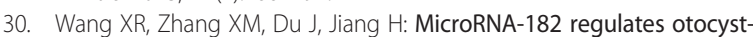
derived cell differentiation and targets T-box1 gene. Hear Res 2012, 286(1-2):55-63.

31. Wang X, Gocek E, Liu CG, Studzinski GP: MicroRNAs181 regulate the expression of p27Kip1 in human myeloid leukemia cells induced to differentiate by 1,25-dihydroxyvitamin D3. Cell Cycle 2009, 8(5):736-741.

32. Li QJ, Chau J, Ebert PJ, Sylvester G, Min H, Liu G, Braich R, Manoharan M, Soutschek J, Skare P, Klein LO, Davis MM, Chen CZ: miR-181a is an intrinsic modulator of T cell sensitivity and selection. Cell 2007, 129(1):147-161.

33. Frucht CS, Santos-Sacchi J, Navaratnam DS: MicroRNA181a plays a key role in hair cell regeneration in the avian auditory epithelium. Neurosci Lett 2011, 493(1-2):44-48.

34. Kazmierczak P, Sakaguchi H, Tokita J, Wilson-Kubalek EM, Milligan RA, Muller U, Kachar B: Cadherin 23 and protocadherin 15 interact to form tip-link filaments in sensory hair cells. Nature 2007, 449(7158):87-91.
35. Ahmed ZM, Riazuddin S, Bernstein SL, Ahmed Z, Khan S, Griffith AJ, Morell RJ, Friedman TB, Riazuddin S, Wilcox ER: Mutations of the protocadherin gene PCDH15 cause Usher syndrome type 1 F. Am J Hum Genet 2001, 69(1):25-34.

36. Ahmed ZM, Riazuddin S, Ahmad J, Bernstein SL, Guo Y, Sabar MF, Sieving P, Riazuddin S, Griffith AJ, Friedman TB, Belyantseva IA, Wilcox ER: PCDH15 is expressed in the neurosensory epithelium of the eye and ear and mutant alleles are responsible for both USH1F and DFNB23. Hum Mol Genet 2003, 12(24):3215-3223.

37. Grimsley-Myers CM, Sipe CW, Geleoc GS, Lu X: The small GTPase Rac1 regulates auditory hair cell morphogenesis. J Neurosci 2009, 29(50):15859-15869.

38. Gentile A, D'Alessandro L, Lazzari L, Martinoglio B, Bertotti A, Mira A, Lanzetti L, Comoglio PM, Medico E: Met-driven invasive growth involves transcriptional regulation of Arhgap12. Oncogene 2008, 27(42):5590-5598

39. Eisen R, Walid S, Ratcliffe DR, Ojakian GK: Regulation of epithelial tubule formation by Rho family GTPases. Am J Physiol Cell Physiol 2006, 290(5):C1297-1309.

40. Jones C, Roper VC, Foucher I, Qian D, Banizs B, Petit C, Yoder BK, Chen P: Ciliary proteins link basal body polarization to planar cell polarity regulation. Nat Genet 2008, 40(1):69-77.

41. Li H, Durbin R: Fast and accurate short read alignment with Burrows-Wheeler transform. Bioinformatics 2009, 25(14):1754-1760.

42. Friedlander MR, Mackowiak SD, Li N, Chen W, Rajewsky N: miRDeep2 accurately identifies known and hundreds of novel microRNA genes in seven animal clades. Nucleic Acids Res 2012, 40(1):37-52.

43. Voorhoeve PM, le Sage C, Schrier M, Gillis AJ, Stoop H, Nagel R, Liu YP, van Duijse J, Drost J, Griekspoor A, Zlotorynski E, Yabuta N, De Vita G, Nojima H, Looijenga LH, Agami R: A genetic screen implicates miRNA-372 and miRNA-373 as oncogenes in testicular germ cell tumors. Adv Exp Med Biol 2007, 604:17-46.

44. Dror AA, Politi Y, Shahin H, Lenz DR, Dossena S, Nofziger C, Fuchs H, Hrabe de Angelis M, Paulmichl M, Weiner S, Avraham KB: Calcium oxalate stone formation in the inner ear as a result of an S/c26a4 mutation. J Biol Chem 2010, 285(28):21724-21735.

doi:10.1186/1471-2164-15-484

Cite this article as: Rudnicki et al:: Next-generation sequencing of small RNAs from inner ear sensory epithelium identifies microRNAs and defines regulatory pathways. BMC Genomics 2014 15:484.

\section{Submit your next manuscript to BioMed Central and take full advantage of:}

- Convenient online submission

- Thorough peer review

- No space constraints or color figure charges

- Immediate publication on acceptance

- Inclusion in PubMed, CAS, Scopus and Google Scholar

- Research which is freely available for redistribution 